\title{
What is the core expertise of the psychiatrist?
}

\author{
Nick Craddock, ${ }^{1}$ Mike Kerr, ${ }^{1}$ Anita Thapar ${ }^{1}$
}

The Psychiatrist (2010), 34, 457-460, doi: 10.1192/pb.bp.110.030114

${ }^{1}$ Department of Psychological Medicine and Neurology, School of Medicine, Cardiff University

Correspondence to Nick Craddock (craddockn@cardiff.ac.uk)
Summary Psychiatrists make important and specific contributions to the care of those with mental health problems and high-quality services should enable patients to benefit optimally from a psychiatrist's distinctive skills. In this article we seek to identify and consider the core expertise of the psychiatrist.

Declaration of interest None.
Recent major changes in organisation and strategic vision of mental health services in the $\mathrm{UK}^{1-3}$ as well as many other countries, have meant that the traditional role of the psychiatrist is changing. It is, however, essential that role changes not be driven purely by legislation, politics or ideology. Rather, changes should make use of a psychiatrist's core expertise, and the special expertise of other professionals, in a way that enhances patient care and ensures quality and safety of services. There has been considerable debate about these issues among UK psychiatrists. $^{4-22}$ A common theme has been the need to define (or perhaps more correctly re-define) the psychiatrist's roles within the context of the shifting service landscape. It has been argued that lack of clarity over the roles of psychiatrists is detrimental to patients ${ }^{19}$ and to recruitment and morale of psychiatrists. ${ }^{18}$

Here, our focus is not the role of the psychiatrist. Rather, we seek to identify and consider what is the core expertise of the psychiatrist. In other words, what are the unique qualities that are brought by a psychiatrist to the clinical team or service. (In marketing terminology, what are the psychiatrist's 'unique selling points'.) Identification and articulation of this expertise may be beneficial for more clearly delineating the optimal roles of psychiatrists within current services and also, crucially, the training needed for, and types of person best suited to become, the psychiatrists who will be effective in future services.

To provide a context for our consideration, we think it is helpful to acknowledge briefly but explicitly four points before we begin. First, service and role changes have varied in timing and nature across psychiatric subspecialties. For example, team working, including distributed roles, was an early feature in child and adolescent mental health services (CAMHS). There has also been substantial variation by geographical location. Second, these issues are not specific to psychiatry and, to a greater or lesser extent, similar changes are now occurring throughout all medical specialties $^{23}$ (e.g. nurse prescribing and extended roles for community pharmacists; the shifting boundaries between general practitioner and secondary care specialist). Third, the changes in service landscape have brought many benefits to mental health including higher priority in health strategy, increased investment and improved access to psychological and social elements of care. Fourth, the changes are underpinned by an irreversible change in public and political thinking and opinion, and psychiatry should embrace, and develop within, this new framework and avoid looking backwards.

We now turn to look at those areas of expertise that can be considered characteristic features of a psychiatrist.

\section{What is unique about psychiatrists?}

By definition, a psychiatrist is distinguished from other mental health professionals by her or his medical training. This is long, encompasses a broad range of knowledge that includes areas relevant to mental and physical health and emphasises pathophysiology and basic sciences. A medical practitioner spends considerable effort learning about human anatomy, physiology and biochemistry in health and sickness, along with basic and clinical pharmacology and the principles of clinical research and evidence evaluation. This is combined with substantial theoretical and practical training in clinically relevant skills such as history taking, physical examination and interpretation of relevant investigations (e.g. laboratory data). Further, during the generic period of training (as medical student and junior doctor) the psychiatrist gains personal experience of primary and secondary healthcare systems across diverse medical specialties, as well as a range of common and rare illnesses and of patients' reactions to them. The medical framework of practice ${ }^{14}$ (certain terms used in this article are explained in Box 1) involves undertaking a systematic assessment (which will almost certainly involve non-medical team members regardless of specialty), making a diagnosis to guide treatment and assess prognosis and critically considering differential diagnoses. Practice is based on using best evidence when it is available to guide decision-making, applying scientific knowledge for guidance, placing the well-being of the patient at the heart of all decisions and being pragmatic and eclectic rather than adhering to one particular school of theory.

The key areas that distinguish psychiatrists from other medical practitioners are their extensive and wide-ranging 
Box 1 Terminology: clearing the minefield before we start

Certain words have become difficult to use because they may trigger instant accusations of paternalism/reductionism or various other pejorative'-isms'. Whether through accidental misunderstanding or political design, the result is to make some topics difficult to discuss with clarity, balance and freedom from unhelpful emotional charge. Here is our attempt to 'make safe' three such terms that we use in this article.

- Medical approach: we use this term for the systematic approach to synthesise and use evidence to guide therapy and advice for an individual who seeks help. We regard it as self-evident that a range of modalities are encompassed in assessment and therapy, consistent with the medical practitioner's broad training. (We do not mean an out-dated, paternalistic or reductionist, narrow biological model.)

- Biology: we use this term to indicate those areas of basic science that traditionally fall within the realm of the life sciences - including physiology, anatomy, biochemistry. It is important to recognise that the distinction between biology and, for instance, psychology (clearly itself a life science) is one of level of understanding, in that any psychological explanation is, in principle, capable of being understood at the level of cellular function. Thus, psychology can be considered as a sub-branch of biology, in the same way that chemistry is a sub-branch of physics.

- Neuroscience: we use this term to refer to the interdisciplinary scientific study of the brain and nervous system, one which includes relevant areas of psychology, philosophy, mathematics, physics and computer science, linguistics, sociology and economics as well as medicine and biology. (This is the accepted modern usage of the term.)

training in clinically relevant aspects of neuroscience ('neuroscience' refers to the wide range of disciplines needed to address the complexities of the nervous system and includes, for example, psychology; Box 1) and the focus on helping patients who present with abnormal thinking, mood and behaviour rather than with uniquely somatic complaints. Although all medical practitioners receive some training in psychological and social aspects of health and disease, psychiatrists spend a major part of their postgraduate training developing an understanding of how these areas integrate with more biological aspects of neuroscience and some psychiatrists become particularly skilled at providing evidence-based psychological and/or social interventions.

From the above it is possible to distil several areas of expertise as core distinguishing attributes of the psychiatrist.

\section{Comprehensive diagnosis}

Psychiatrists are the mental health professionals who have been trained in the process of clinical assessment and diagnosis and who have in-depth knowledge of the broad range of domains that may be relevant. They are trained in the diagnosis of mental disorders and somatic illness. As we will explain later, it is crucial that these are not separated or considered as mutually exclusive sets of problems. At one extreme, variations in thinking, mood and behaviour can be normal features of life within the context of age, development and psychological and social circumstances. At the other extreme, they may be profoundly abnormal states that threaten life and may reflect serious abnormalities in the physical, psychological, developmental or social state of the individual. Taking a systematic history, making observations, noting and being alert to the presence and relevance of physical health problems as well as synthesising information from different sources (verbal, observation, physical examination and appropriate investigation) are crucial to making the informed decisions that can launch a patient on the most appropriate therapeutic path at the earliest opportunity.

\section{Somatic illness}

The common co-occurrence of somatic disease and mental ill health is increasingly recognised. ${ }^{24,25}$ Physical illnesses can initially present with psychiatric symptoms. It is essential that these not be missed. Many neuropsychiatric disorders are commonly accompanied by problems that are viewed as somatic (e.g. autism and epilepsy) and others substantially increase the risk of somatic health problems (e.g. depression and cardiovascular disease). Further, treatments for somatic illness often have effects on mental well-being and treatments for mental illness often have somatic effects. Substance misuse is a case in point substance problems may lead to somatic and psychiatric disorders as a result of intoxication, dependence and withdrawal. Psychiatrists have the training to evaluate, understand and manage these sometimes complicated, overlapping presentations.

\section{Biology and clinically relevant aspects of neuroscience}

As we discuss in our 'terminology minefield' (Box 1), the supposed neat distinction between 'biological', 'psychological' and 'social' does not stand up to the scrutiny of modern science. ${ }^{20,26}$ Rather, they are different levels or frameworks to think about and understand the contributions to illness and possible approaches to treatment. However, with that caveat in mind, the psychiatrist has considerable training in the basic disciplines of biology and their interplay with psychosocial contexts. Thus she or he is well trained in using a broad scientific framework to understand causal risk factors and their complex inter-relationships, consider useful ways of categorising disorders, provide the rationale for particular treatments, select appropriate psychotropic medication when needed, balance the risks and benefits of such treatments and predict clinical outcomes. As clinical neuroscience increases the understanding of mental illness (and its relationships with somatic illness) and new treatments become available, this is likely to become increasingly important. ${ }^{14}$ Thus, a psychiatrist is the mental health professional with the background and training to apply the full breadth of relevant scientific knowledge to help those with mental illness. 


\section{Leadership, problem solving and pragmatism}

A major criterion in selecting students to train in medicine is strong academic ability, motivation and vocational commitment. Their training is broad, deep and intense and encompasses somatic and mental health problems. The combination of academic ability and breadth and depth of training should equip all doctors, including psychiatrists, to lead teams, manage clinical complexity in a pragmatic and flexible manner and consider a range of diagnostic and therapeutic possibilities. It is, however, worth noting that dealing with complex clinical cases effectively requires experience of managing more straightforward cases. ${ }^{16}$ Psychiatrists are especially able to make balanced and informed judgements about when to depart from the plethora of management protocols and guidelines. This is an extremely important element of high-quality care because the complexity of mental illness and the enormous individual variation among patients mean their needs are not always best served by rigid adherence to pre-specified consensus guidelines. In particular, psychiatrists are equipped to coordinate and oversee situations where treatment involves multiple modalities (e.g. cognitive-behavioural therapy and antidepressants; or concurrent treatment for both psychiatric and somatic illness). Thinking beyond the individual patient situation, psychiatrists can usefully bring their problem-solving skills to a range of situations within mental health services, including service planning, development and evaluation as well as team management. The psychiatrists' training also makes them effective and valuable interfaces with non-psychiatric medical practitioners, including general practitioners, paediatricians and physicians.

\section{Research}

There is a strong scientific component to general medical and specialist psychiatric training, which encompasses a wide variety of disciplines relevant to understanding human health and disease. There is also a strong culture and tradition of research and enquiry. It is extremely important for future improvement in understanding causation, mechanisms and treating mental illness that mental health professionals as well as basic scientists are active in high-quality research. Psychiatrists are particularly well placed to provide the overview and perspective needed to bridge the interface across clinical and basic research that is increasingly complex and cross-disciplinary. This includes formulating new research questions based on clinical insights. Furthermore, the psychiatrist's training allows for critical analysis of research. This will be crucial as our evidence base expands.

\section{Advocacy}

Psychiatrists are able to act as a voice within the medical profession for the importance of psychological and social approaches and as a voice among mental health professionals for the importance of taking account of somatic illness, biological factors and, when appropriate, a medical approach. This includes influencing medical school curricula and postgraduate medical training to ensure that all doctors have a balanced and accurate understanding of mental illness and the important role of psychiatry for human health. Psychiatrists are able to act as strong and authoritative advocates for, and leaders of, service developments that are evidence-based, of high quality and address the breadth of needs of those who use services. On a personal level, the psychiatrist is able to act as advocate for, and perhaps broker of, interventions across multiple therapeutic domains for an individual patient.

\section{Adaptability}

An extremely important component of the psychiatrist's skills is flexibility to deal with changes in understanding of the aetiology and treatment of psychiatric illness as well as variations in service configuration. Having a broad range of expertise means the psychiatrist does not need to be wedded to one intervention - such as a pharmacological or psychotherapeutic intervention, or just one understanding of mechanisms - such as genetic or social. Equally, this flexibility allows a psychiatrist to have joint roles, for instance combining management, teaching and/or research with service provision.

\section{The role of the psychiatrist}

The role of the psychiatrist must inevitably vary over time and place according to the specific service model, statutory legal frameworks, needs of the population being served and the number and skill mix of other professionals. Although this article does not seek to define the role of the psychiatrist, consideration of the core skills of the psychiatrist (Box 2) should help ensure that those skills are matched to their role in a way that maximises benefits to the individual patient and to the service. Such matching and clarity may also help in enhancing psychiatric professional identity, encouraging recruitment and increasing morale and job satisfaction.

\section{Box 2 Core attributes of the psychiatrist}

- Uses broad perspective including biological, psychological and social factors

- Uses comprehensive and systematic diagnosis of both mental and somatic ill health

- Has knowledge of a broad range of diseases

- Uses broad background in biology and clinically relevant aspects of neuroscience

- Is willing and able to provide clinical leadership

- Embraces complexity and uses pragmatism

- Values research and uses evidence

- Acts as advocate for individuals with mental illness including championing high-quality services

- Uses broad training to adapt as knowledge develops 


\section{Conclusions}

We have identified several areas that can be considered the core attributes of the psychiatrist (Box 2) and have touched on some implications in relation to the role of the psychiatrist. A recent leader article in the journal World Psychiatry posed the question, 'Are psychiatrists an endangered species?'27 There are certainly many 'species' of mental health professional competing within the same habitat. It will be necessary for the psychiatric profession to adapt to the changing environment by maintaining and developing their optimal qualities and passing these to the future generations of psychiatrists. ${ }^{28}$ This should ensure that, two centuries after Reil first described the specialty of psychiatry, ${ }^{29,30}$ patients can continue to benefit from the particular expertise and training of medical practitioners, who specialise in psychiatric illness and use their broad medical and biological expertise and diagnostic skills effectively within the context of an appreciation of the psychosocial factors and full range of available treatment modalities. $^{31}$

\section{Acknowledgements}

We are grateful for helpful discussions and/or comments on drafts of the manuscript to: Dr Bridget Craddock, Professor Ilana Crome, Dr Martin Gee, Dr Glyn Jones, Dr Antonio Munoz, Dr Jon Van Niekerk, Dr Clare Oakley, Dr Rob Potter, Dr Oliver White and Dr Ajay Thapar.

\section{About the authors}

Nick Craddock is Professor of Psychiatry and Honorary Consultant Psychiatrist, Mike Kerr is Professor of Learning Disability Psychiatry and Honorary Consultant Psychiatrist, Anita Thapar is Professor of Child and Adolescent Psychiatry and Honorary Consultant Psychiatrist, Department of Psychological Medicine and Neurology, School of Medicine, Cardiff University, UK.

\section{References}

1 Department of Health. National Service Framework for Mental health: Modern Standards and Service Models. Department of Health, 1999 (http://www.dh.gov.uk/en/Publicationsandstatistics/Publications/ PublicationsPolicyAndGuidance/DH_4009598).

2 Royal College of Psychiatrists, National Institute for Mental Health in England, Changing Workforce Programme. New Ways of Working for Psychiatrists: Enhancing Effective, Person-Centred Services through New Ways of Working in Multidisciplinary and Multiagency Contexts. Final Report 'But Not the End of the Story'. Department of Health, 2005.

3 Deparment of Health. New Horizons: Towards a Shared Vision for Mental Health - Report on Responses to the Consultation. Deparment of Health, 2009 (http://webarchive.nationalarchives.gov.uk/+/www. dh.gov.uk/ en/Consultations/Responsestoconsultations/DH_114076).

4 Royal College of Psychiatrists. The Responsibilities of Consultant Psychiatrists, Revised Statement (Council Report CR51). Royal College of Psychiatrists, 1996

5 Royal College of Psychiatrists. Roles and Responsibilities of a Consultant in General Psychiatry (Council Report CR94). Royal College of Psychiatrists, 2001.

6 Royal College of Psychiatrists. Consultants as Partners in Care: The Roles and Responsibilities of Consultant Psychiatrists in the Planning and Provision of Mental Health Services for People with Severe Mental IIIness (Council Report (R96). Royal College of Psychiatrists, 2001.
7 Royal College of Psychiatrists. Role of Consultants with Responsibility for Substance Misuse (Addiction Psychiatrists): Position Statement by the Faculty of Substance Misuse (Council Report CR97). Royal College of Psychiatrists, 2001.

8 Royal College of Psychiatrists. Role and Contribution of the Consultant Psychiatrist in Psychotherapy in the NHS (Council Report CR98). Royal College of Psychiatrists.

9 Royal College of Psychiatrists, Royal College of General Practitioners. Roles and Responsibilities of Doctors in the Provision of Treatment for Drug and Alcohol Misusers (Council Report CR131). Royal College of Psychiatrists, 2005.

10 Royal College of Psychiatrists. Role of the Consultant Psychiatrist in Psychotherapy (Council Report CR139). Royal College of Psychiatrists, 2006.

11 Royal College of Psychiatrists. Roles and Responsibilities of the Consultant in General Adult Psychiatry (Council Report CR140). Royal College of Psychiatrists, 2006.

12 Royal College of Psychiatrists. Role of the Consultant Psychiatrist: Leadership and Excellence in Mental Health (Occasional Paper OP74). Royal College of Psychiatrists, 2010.

13 Anonymous. Molecules and minds. Lancet 1994; 343: 681-2.

14 Kendell RE. The next 25 years. Br J Psychiatry 2000; 176: 6-9.

15 Shah $\mathrm{P}$, Mountain D. The medical model is dead - long live the medical model. Br J Psychiatry 2007; 191: 375-7.

16 Malik A, White O, Mitchell J, Henderson P, Oakley C. New Ways of Working and psychiatric trainees. Psychiatr Bull 2008; 32: 230-2.

17 Gee M. New Ways of Working threatens the future of the psychiatric profession. Psychiatr Bull 2007; 31: 315.

18 Gee M. New Ways of Working: time to abandon the phrase. Psychiatr Bull 2008; 32: 233.

19 Craddock N, Antebi D, Attenburrow MJ, Bailey A, Carson A, Cowen P, et al. Wake-up call for British psychiatry. Br J Psychiatry 2008; 193: 6-9.

20 Bullmore $\mathrm{E}$, Fletcher $\mathrm{P}$, Jones $\mathrm{PB}$. Why psychiatry can't afford to be neurophobic. Br J Psychiatry 2009; 194: 293-5.

21 Hawley C, Drummond L, Knight J. NHS psychiatry: the need for constructive debate. Invited commentary on ... The trouble with NHS psychiatry in England. Psychiatric Bull 2009; 33: 299-302.

22 Gee M. Psychiatry over the next decade: the return of common sense. Midlands Med 2010; 26: 17-9.

23 NHS Employers. The Role of the Doctor Consensus Statement. NHS Employers (http://www.nhsemployers.org/PlanningYourWorkforce/ MedicalWorkforce/Future-of-the-medical-workforce/Pages/Role-ofdoctor-consensus.aspx).

24 Leucht S, Fountoulakis K. Improvement of the physical health of people with mental illness. Curr Opin Psychiatry 2006; 19: 411-2.

25 Prince M, Patel V, Saxena S, Maj M, Maselko J, Phillips MR, et al. No health without mental health. Lancet 2007; 370: 859-77.

26 Kendell RE. The distinction between mental and physical illness. Br J Psychiatry 2001; 178: 490-3.

27 Katschnig $\mathrm{H}$. Are psychiatrists an endangered species? Observations on internal and external challenges to the profession. World Psychiatry 2010; 9: 21-8

28 Craddock N, Craddock B. Patients must be able to derive maximum benefit from a psychiatrist's medical skills and broad training. World Psychiatry 2010; 9: 30-1.

29 Reil J, Hoffbauer J. Beyträge zur Beförderung einer Kurmethode auf psychischem Wege. [Contributions to the Advancement of a Treatment Method by Psychic Ways.] Curt'sche Buchhandlung, 1808.

30 Marneros A. Psychiatry's 200th birthday. Br J Psychiatry 2008; 193: 1-3.

31 Craddock N. A psychiatrist is . . . - in 100 words. Br J Psychiatry 2010; 196: 473 . 